\title{
A single-center retrospective observational cohort study on postoperative pain management: better with single or continuous doses of opiates or acetaminophen?
}

Takashi Tennichi ( $\nabla$ takashi25.ten@gmail.com )

Kanazawa University https://orcid.org/0000-0001-5728-6183

Takumi Taniguchi

Kanazawa University

Research article

Keywords: Continuous administration, single administration, opioid, delirium, Intensive Care Delirium Screening Checklist, Numerical Rating Scale

Posted Date: July 9th, 2019

DOl: https://doi.org/10.21203/rs.2.11092/v1

License: (c) (i) This work is licensed under a Creative Commons Attribution 4.0 International License.

Read Full License 


\section{Abstract}

Background: Proper pain management after cardiac surgery is essential for the prevention of postoperative complications, and several protocols have been proposed in this regard. The continuous administration of an opioid is a common approach now because it allows for more prolonged postsurgical analgesic effects. However, this method has several problems, such as the incidence of protracted delirium, nausea, and vomiting. The present study aims to evaluate the effects of two opioid administration methods on postoperative pain management and other clinical outcomes after cardiac surgery. Methods: This is a retrospective observational cohort study that enrolled 184 adult patients who underwent elective cardiac surgery. After ICU admission, patients were divided into two groups: 101 patients who received a single administration of fentanyl ( $25 \mathrm{mg}$ boluses and a 30 min lockout time) and an administration of acetaminophen ( $15 \mathrm{mg} \mathrm{kg}-1$ and a 6 hour lockout time) [group S] and 83 patients who underwent continuous administration of fentanyl ( $25 \mathrm{mg}$ hour- 1 with $35 \mathrm{mg}$ boluses and a $30 \mathrm{~min}$ lockout time) and an administration of acetaminophen ( $15 \mathrm{mg} \mathrm{kg}-1$ and a 6 hour lockout time) [group C]. For all patients, the Intensive Care Delirium Screening Checklist (ICDSC) scores were measured soon after extubation (0 hours) and 12, 24, and 36 hours later. Similarly, for all patients, NRS scores were also quickly measured after extubation (0 hours) and 8, 16, and 24 hours later. Results: ICDSC scores in group $S$ were significantly lower than those in group $C 0$ and 12 hours after extubation $(p<0.001)$. However, ICDSC scores 24 and 36 hours post extubation did not differ significantly between the two groups. NRS scores did not differ significantly between the two groups until 24 hours after extubation. Conclusions: Compared with single administration of fentanyl with acute administration of acetaminophen, continuous administration of postoperative fentanyl with acute administration of acetaminophen may lead to increased risk for postoperative delirium. Therefore, acute administration of fentanyl may be preferable for continuous use in postoperative cardiac surgery patients.

\section{Background}

Postoperative pain relief is a major medical challenge. Patient experiences following surgery have been investigated in many countries, including France, Germany, Italy, the Netherlands, United Kingdom and the USA, and up to $80 \%$ of those surveyed experienced postoperative pain [1-8]. Poorly managed postoperative pain, especially following cardiac surgery, can also increase postoperative complication rates and impair recovery [9]. Proper pain control after cardiac surgery is thus essential because it can facilitate a reduction in postoperative complications [10].

Several treatments have been proposed for postoperative pain management, and these include the continuous administration of opioids, thoracic epidural analgesia, and paravertebral blocks [11]. Continuous administration of opioid is more common than thoracic epidural analgesia and paravertebral block because of the bleeding associated with heparin administration after cardiac surgery. Walder et al. found that the continuous administration of opioids reduced postoperative pulmonary complications [12]. However, several reports have indicated that the incidence of prolonged delirium is higher when an opioid is administered than when it is not $[13,14]$. Pisani et al. [15] reported that continuous 
administration of an opioid was associated with persistent delirium in older intensive care unit (ICU) patients. Nishino et al. [16] similarly found that the use of opioids should not be advocated because of the risk of analgesia-related complications, although continuous administration of opioids allows for more prolonged post-surgical analgesic effects.

These earlier studies reveal that the net effectiveness of the continuous administration of opioids after cardiac surgery remains unclear. We hypothesized that a single administration of opioids would provide better postoperative management, including pain, compared to a continuous administration of opioids following cardiac surgery. Therefore, we retrospectively evaluated the effects of two opioid administration methods on postoperative pain management and other clinical outcomes after cardiac surgery.

\section{Methods}

Ethical approval for the present study (approval number: 2632) was given by the Ethical Committee of the Institutional Review Board of the Kanazawa University School of Medicine, Kanazawa, Japan on November 3, 2017. The present retrospective observational cohort study was conducted at Kanazawa University Hospital between November 2016 and March 2018. As the present study involved a retrospective analysis of in-patient charts and anesthetic report data from a concurrently recorded computerized database, the Ethics Committee of the Kanazawa University Hospital waived the need for informed consent. We will instead release information regarding the present study to the public on an optout basis.

\section{Incision criteria}

Admitted into the present study were patients undergoing cardiac surgery procedures, such as coronary artery bypass grafting (CABG), heart valve surgeries, and cooperations on the aorta through a primary full median sternotomy. We included patients with an American Society of Anesthesiologists physical status (ASA-PS) of under 3.

\section{Exclusion criteria}

We did not include patients with any of the following characteristics: an ASA-PS of 4 or 5 , a Glasgow Coma Scale score $<15$, history of or current dialysis, and severe liver damage (Child-Pugh grade of B or C). We also excluded patients with use of a preoperative opioid, a central nervous depressant (phenothiazine or barbiturate), a narcotic antagonist analgesic (buprenorphine or pentazocine), a 
monoamine oxidase inhibitor, a tricyclic antidepressant, or an antihistamine agent. Furthermore, patients who underwent emergency surgery or surgery without a median sternotomy were excluded.

\section{Anesthesia Procedure}

Anesthesia was induced with midazolam and fentanyl over a $60 \mathrm{~s}$ period. After rocuronium was administered for muscle relaxation, tracheal intubation was performed. Anesthesia was then maintained with fentanyl and/or remifentanil, sevoflurane and/or propofol infusion. Five-lead ECG, pulse oximetry, invasive arterial and pulmonary artery pressure and bispectral index values through IntelliVue MX800 ${ }^{\mathrm{TM}}$ (Royal Philips, Amsterdam, Netherlands) were also monitored. The volume of fluids, the number of transfusions, and the doses of vasopressors administered during the operation were calculated from medical records. Immediately after the operation, all patients were moved to the ICU. They were then extubated after demonstrating functional recovery and hemodynamic and respiratory stability, as determined by their respective attending physicians.

In the present study, patients were divided into two groups based on the pain management each received. One group (group S) received a single administration of fentanyl ( $25 \mathrm{mg}$ boluses and $30 \mathrm{~min}$ lockout time) and an administration of acetaminophen ( $15 \mathrm{mg} \mathrm{kg}^{-1}$ and 6 hour lockout time), and the other (group C) underwent a continuous administration of fentanyl $\left(25 \mathrm{mg}\right.$ hour ${ }^{-1}$ with $35 \mathrm{mg}$ boluses and a 30 min lockout time) and an administration of acetaminophen ( $15 \mathrm{mg} \mathrm{kg}^{-1}$ and 6 hour lockout time) (Table S1, Additional file 1).

As our primary outcome metric, we used the Intensive Care Delirium Screening Checklist (ICDSC) scores 12 hours after extubation. Secondary outcomes included the following: total doses of dexmedetomidine and propofol; time to extubation; a total post-surgical dose of fentanyl; total time of continuous administration of fentanyl; total doses of acetaminophen; NRS scores 8 hours after extubation; oxygenation ( $\mathrm{PaO}_{2} / \mathrm{FiO}_{2}(\mathrm{P} / \mathrm{F})$ ratio) after extubation; an incidence of postoperative nausea and vomiting (PONV) and constipation; postoperative hepatic (aspartate aminotransferase (AST), alanine aminotransferase (ALT)) and renal (serum creatinine) functioning; and a total length of ICU stay.

Nurses evaluated the ICDSC and numerical rating scale (NRS) scores in the ICU. PONV was defined as the occurrence of at least one episode of nausea or vomiting within the first 24 hours after extubation, where vomiting was defined as the expulsion of gastric content. Constipation was defined as a failure of the bowel to open for three consecutive days [17]. Nurses in the ICU monitored patient symptoms and collected these data. The AST, ALT, serum creatinine, the P/F ratio, and the total length of ICU stay were collected from the electronic chart of each subject. 
Continuous data are described as means \pm standard deviations. Categorical variables are presented as numbers and percentages. The Student's $t$-test for continuous variables and the chi-square or Fisher's exact test for categorical variables were used to compare the baseline of the two groups. The ICDSC scores $(0,12,24$, and 36 hours after extubation), as well as the NRS scores $(0,8,16$, and 24 hours after extubation) across time points, were compared between the two groups using a repeated-measures analysis of variance. $p$-Values $<0.05$ were considered statistically significant. The Statistical Package for the Social Sciences (Version 23, IBM Inc., Armonk, NY, USA) was used for all analyses.

\section{Results}

Figure 1 is a flowchart of patients included in the present study, for which 201 individuals were assessed for eligibility. Seventeen subjects were not enrolled: 3 because of aborting surgery and 14 because of our exclusion criteria. More specifically, these are the reasons for excluding these patients: 2 because of an ASA-PS of IV, 7 because of a history of or current dialysis, and 5 because of surgery without a median sternotomy. In total, 184 patients were included, and 101 were treated with a single administration of fentanyl or an infusion of acetaminophen (group S), and 83 received a continuous administration of fentanyl (group C).

Table 1 presents the preoperative and operative patients' data. The mean age was 76 years $( \pm 11)$, and $73 \%$ of our subjects were male. Most common comorbidities were hypertension (45\%), hyperlipidemia $(32 \%)$ and diabetes mellitus (12\%). All patients were classified as ASA-PS 3 . The distribution of the operative procedures was coronary artery bypass grafting (CABG) (35\%), heart valve surgeries (53\%), and cooperations on the aorta $(12 \%)$.

Table 2 presents differences in the intraoperative profiles of patients. The intraoperative fentanyl dose did not differ significantly between the two groups $(27.8 \pm 10.1$ vs. $28.9 \pm 12.1, p=.494)$. The volume of transfusion, including RCC, FFP, and PC, also did not differ significantly between the two groups.

Figure 2 illustrates the change in ICDSC scores after ICU admission. These scores were significantly lower in group $S$ than in group $C 0$ and at 12 hours after extubation $(p<0.001)$. However, the ICDSC scores 24 and 36 hours post extubation did not differ significantly between the two groups.

The total dose of fentanyl was lower in group $S(p<0.0001)$ than in group $C$, and the total dose of acetaminophen in group $S$ was higher than that in group $C(p<0.0001)$.

Table 3 presents the postoperative profile of the patients. The total dose and duration of propofol and dexmedetomidine administrations did not differ significantly between the two groups. However, the time to extubation in group $S$ was shorter than that in group $C(p=0.007)$. Post-extubation postoperative hepatic (AST and ALT) and renal (serum creatinine) functions, as well as oxygenation (P/F ratio), also did 
not differ between the two groups; neither did the occurrence of constipation. PONV in group S was significantly lower than that in group $C(p<0.001)$, and the length of ICU stay in group $S$ was shorter than that in group $C(p=0.003)$.

Figure 3 illustrates changes in the NRS scores after ICU admission. We found that these scores did not differ significantly between the two groups $0,8,16$, and 24 hours after extubation.

\section{Discussion}

The ICDSC score of group S was lower than that of group $\mathrm{C}$ after extubation, regardless of the equivalent sedation level. Time to extubation and length of ICU stay of group S were also shorter than those of group $\mathrm{C}$, and the PONV incidence in group $\mathrm{S}$ was lower than that in group $\mathrm{C}$.

The total fentanyl dose was lower in S group than in group C, but the total dose of acetaminophen was higher in group $S$ than in group $C$. There were no significant differences in NRS scores between both groups regardless of whether through a continuous or a single administration of fentanyl; this is consistent with previous study results indicating that postoperative pain control did not differ between intravenous acetaminophen administration and the continuous infusion of fentanyl $[18,19]$. Mohammad et al. [20] also reported that intravenous acetaminophen was better for controlling postoperative pain than continuous intravenous fentanyl was. Thus, these findings indicate that a single administration of fentanyl and a non-opioid analgesic is enough to control postoperative pain after cardiac surgery.

Opioid drugs are widely used as moderate to strong analgesic agents [21] and are thus a mainstay of an effective analgesia strategy after surgery for the management of moderate to severe pain [22]. Although it may be assumed that the continuous administration of opioids maintains more consistent blood opioid levels, and thus more stable analgesic effects, than single administration does, these findings have not been confirmed with the appropriate studies. Despite this gap in the literature, it is nonetheless clear that the continuous administration of opioids contributes more to the incidence of delirium than acute opioid or acetaminophen administration does. Jie et al. [23] reported that continuous administration of fentanyl also increased the risk of delirium. Our study also reveals that opioid use leads, in some cases, to an increased incidence of adverse effects such as PONV. Hence, these findings suggest that it is necessary to correctly administer analgesic agents on a case-by-case basis without regarding the continuous administration of opioids as a default alternative.

It is well known that postoperative pain adversely affects physical functioning, recovery, and quality of life [24-26], which in turn often drive one another, leading to a broad variety of postoperative complications such as dysfunction of the nervous, respiratory, circulatory, gastrointestinal, and hematic systems, as well as infection. Careful postoperative pain management is, therefore, essential, and advances in this area can be assisted by studies, such as ours, that examine multiple treatment modalities and methods for pain management. A multimodal approach to analgesia is also currently 
recommended for use in post-surgical patients [12]. Controlling postoperative pain with the lowest possible doses of opioids is certainly a high priority.

Although the present study contributes significantly to the literature, it also has several critical limitations that warrant discussion. First, we utilized only the ICDSC as a metric to assess postoperative delirium. However, additional instruments, such as the Confusion Assessment Method for the Intensive Care Unit (CAM-ICU) [27], could also have been used. However, this may not have been necessary because there are no documented differences between the ICDSC and CAM-ICU in these instruments' assessments of postoperative pain [28]. Second, patients in our study were administered acetaminophen and fentanyl. Therefore, associations between postoperative delirium and acetaminophen, as well as other agents, should be considered both in our results and in future work. Finally, the present study utilized a retrospective, observational design in which the attending physician decided whether and when to wake and extubate each patient. No clear and consistently applied criteria were used in this regard; this is a critical limitation. Future multicenter, randomized clinical trials are needed to complement the pilot data demonstrated in our present, single center, nonrandomized study.

\section{Conclusions}

The continuous administration of postoperative fentanyl may cause an increased postoperative delirium risk. Combination of acute administration of fentanyl and acetaminophen may be preferable because acetaminophen contributes to the reduction of the total dose of fentanyl and the frequency of postoperative delirium in postoperative cardiac surgery patients.

\section{Abbreviations}

ICU: intensive care unit

CABG: coronary artery bypass grafting

ASA-PS: American Society of Anesthesiologists Physical Status

ICDSC: Intensive Care Delirium Screening Checklist

P/F: $\mathrm{PaO}_{2} / \mathrm{FiO}_{2}$

$\mathrm{PaO}_{2}$ : arterial oxygen tension

$\mathrm{FiO}_{2}$ : inspired oxygen fraction

PONV: Postoperative nausea and vomiting

ALT: aspartate aminotransferase

AST: alanine aminotransferase 
NRS: Numerical Rating Scale

CAM-ICU: Confusion Assessment Method for the Intensive Care Unit

\section{Declarations}

Ethics approval and consent to participate. Ethical approval for the present study (approval number: 2632) was given by the Ethics Committee of the Institutional Review Board of the Kanazawa University School of Medicine, Kanazawa, Japan on November 3, 2017. The present retrospective observational cohort study was conducted at Kanazawa University Hospital between November 2016 and March 2018. As the present study involved a retrospective analysis of in-patient charts and anesthetic report data from a concurrently recorded computerized database, the Ethics Committee of the Kanazawa University Hospital waived the need for informed consent. We will release information regarding the present study to the public on an opt-out basis instead.

Consent for publication. Not applicable.

Availability of data and materials. The datasets used and analyzed during the current study are available from the corresponding author on reasonable request.

Competing interests. The authors declare that they have no competing interests.

Funding. Not applicable.

Authors' contributions. Takashi T. conceived and coordinated the study, designed it, carried out the data collection and analysis, and wrote the paper. Takumi T. revised the paper. Both authors reviewed the results and approved the final version of the manuscript.

Acknowledgments. The authors would like to thank Enago (www.enago.jp) for the English language review.

\section{References}

1. Fletcher D, Fermanian C, Mardaye A, Aegerter P. Pain and Regional Anesthesia Committee of the French Anesthesia and Intensive Care Society (SFAR). A patient-based national survey on postoperative pain management in France reveals significant achievements and persistent challenges. Pain. 2008;137:441-51.

2. Maier C, Nestler N, Richter H, Hardinghaus W, Pogatzki-Zahn E, Zenz M, et al. The quality of pain management in German hospitals. Dtsch Arztebl Int. 2010;107:607-14.

3. Coluzzi F, Mattia C, Savoia G, Clemenzi P, Melotti R, Raffa RB, et al. Postoperative Pain Surveys in Italy from 2006 and 2012: (POPSI and POPSI-2). Eur Rev Med Pharmacol Sci. 2015;19:4261-9. 
4. Sommer M, de Rijke JM, van Kleef M, Kessels AG, Peters ML, Geurts JW, et al. The prevalence of postoperative pain in a sample of 1490 surgical inpatients. Eur J Anaesthesiol. 2008;25:267-74.

5. Hamilton L, Macgregor A, Warmington V, Pinch E, Gaffney K. The prevalence of inflammatory back pain in a UK primary care population. Rheumatology (Oxford). 2014;53:161-4.

6. Apfelbaum JL, Chen C, Mehta SS, Gan TJ. Postoperative pain experience: results from a national survey suggest postoperative pain continues to be undermanaged. Anesth Analg. 2003;97:534-40.

7. Gan TJ, Habib AS, Miller TE, White W, Apfelbaum JL. Incidence, patient satisfaction, and perceptions of post-surgical pain: results from a US national survey. Curr Med Res Opin. 2014;30:149-60.

8. Meißner W, Komann M, Erlenwein J, Stamer U, Scherag A. The quality of postoperative pain therapy in German hospitals. Dtsch Arztebl Int. 2017;114:161-7

9. Rawal N. Current issues in postoperative pain management. Eur J Anaesthesiol. 2016;33:160-71.

10. Pennefather SH, McKevith J. Pain management after thoracic surgery. In: Slinger P., editor. Principles and practice of anesthesia for thoracic surgery. Springer Science+Business Media; 2011. p. 675-707.

11. Garimella V, Cellini C. Postoperative pain control. Clin Colon Rectal Surg. 2013;26:191-6.

12. Walder B, Schafer M, Henzi I, Tramer MR. Efficacy and safety of patient-controlled opioid analgesia for acute postoperative pain: a quantitative systematic review. Acta Anaesthesiol Scand. 2001;45:795-804.

13. Devlin JW, Skrobik Y, Gélinas C, Needham DM, Slooter AJC, Pandharipande PP, et al. Clinical Practice Guidelines for the prevention and management of pain, agitation/sedation, delirium, immobility, and sleep disruption in adult patients in the ICU. Crit Care Med. 2018;46:e825-73.

14. Heo DY, Hwang BM. Intravenous patient-controlled analgesia has a positive effect on the prognosis of delirium in patients undergoing orthopedic surgery. Korean J Pain. 2014;27:271-7.

15. Pisani MA, Murphy TE, Araujo KL, Slattum P, Van Ness PH, Inouye SK. Benzodiazepine and opioid use and the duration of intensive care unit delirium in an older population. Crit Care Med. 2009;37:177-83.

16. Nishio S, Fukunishi S, Juichi M, Koyanagi S, Fujihara Y, Fukui T, et al. Comparison of continuous femoral nerve block, caudal epidural block, and intravenous patient-controlled analgesia in pain control after total hip arthroplasty: a prospective randomized study. Orthopedic Review. 2014;6:5138.

17. Mostafa SM, Bhandari S, Ritchie G, Gratton N, Wenstone R. Constipation and its implications in the critically ill patient. Br J Anaesth. 2003;91:815-9.

18. Go M, Abe N, Naritani T, Minato F, Kato M, Ritsuko GO. Efficacy of routine intravenous acetaminophen compared with continuous infusion of fentanyl for postoperative pain management after laparoscopic inguinal hernia repair. Tokushima Red Cross Hospital Medical Journal. 2018;23:22-8.

19. Kouchek M, Mansouri B, Mokhtari M, Goharani R, Miri MM, Sistanizada M. A comparative study of intravenous paracetamol and fentanyl for pain management in ICU. Iran J Pharm Res. 2013;12:1938. 
20. Zolhavarieh SM, Mousavi-Bahar SH, Mohseni M, Emam AH, Poorolajal J, Majzoubi F. Effect of intravenous acetaminophen versus fentanyl on postoperative pain after transurethral lithotripsy. Rev Bras Anestesiol. 2019;69:131-6

21. Trivedi M, Shaikh S, Gwinnut C. Pharmacology of opioids. Update in Anesthesia. 2007;118-24.

22. Coluzzi F, Bifulco F, Cuomo A, Dauri M, Leonardi C, Melotti RM, et al. The challenge of perioperative pain management in opioid-tolerant patients. Ther Clin Risk Manag. 2017;13:1163-73.

23. Yang J, Zhou Y, Kang Y, Xu B, Wang P, Lv Y, et al. Risk factors of delirium in sequential sedation patients in intensive care units. Biomed Res Int. 2017;2017:3539872.

24. VanDenKerkhof EG, Hopman WM, Reitsma ML, Goldstein DH, Wilson RA, Belliveau P, et al. Chronic pain, healthcare utilization, and quality of life following gastrointestinal surgery. Can J Anaesth. 2012;59:670-80.

25. Peters ML, Sommer M, de Rijke JM, Kessels F, Heineman E, Patijn J, et al. Somatic and psychologic predictors of long-term unfavorable outcome after surgical intervention. Ann Surg. 2007;245:487-94.

26. Wu CL, Naqibuddin M, Rowlingson AJ, Lietman SA, Jermyn RM, Fleisher LA. The effect of pain on health-related quality of life in the immediate postoperative period. Anesth Analg. 2003;97:1078-85.

27. Khan BA, Perkins AJ, Gao S, Hui SL, Campbell NL, Farber MO, et al. The CAM-ICU-7 delirium severity scale: a novel delirium severity instrument for use in the intensive care unit. Crit Care Med. 2017;45:851-7.

28. Chanques G, Ely EW, Garnier O, Perrigault F, Eloi A, Carr J, et al. The 2014 updated version of the Confusion Assessment Method for the Intensive Care Unit compared to the 5th version of the Diagnostic and Statistical Manual of Mental Disorders and other current methods used by intensivists. Ann Intensive Care. 2018;8:33.

\section{Tables}

Table 1 Preoperative and operative patients' data 


\begin{tabular}{lccc}
\hline & S group (n:101) & C group (n:83) & p value \\
\hline Gender (M/F) & $76 / 25$ & $59 / 24$ & .616 \\
Age (yr) & $67.8 \pm 13.3$ & $69.7 \pm 9.4$ & .268 \\
Height (cm) & $161.1 \pm 13.0$ & $161.8 \pm 9.4$ & .668 \\
Weight (kg) & $61.7 \pm 14.9$ & $62.5 \pm 12.7$ & .695 \\
Hypertension n (\%) & $44(43.6 \%)$ & $39(46.9 \%)$ & .658 \\
Diabetes n (\%) & $15(14.9 \%)$ & $9(10.8 \%)$ & .512 \\
Hyperlipidemia n (\%) & $32(31.7 \%)$ & $28(33.7 \%)$ & .875 \\
Asthma n (\%) & $4(4.0 \%)$ & $5(6.0 \%)$ & .734 \\
History of stroke n (\%) & $10(9.9 \%)$ & $11(13.2)$ & .494 \\
AST (unit/L) & $29.9 \pm 13.3$ & $28.8 \pm 14.7$ & .604 \\
ALT (unit/L) & $18.0 \pm 7.4$ & $19.7 \pm 8.3$ & .141 \\
Serum creatinine (mg/dL) & $0.90 \pm 0.59$ & $0.88 \pm 0.42$ & .791 \\
EF (\%) & $10(9.9 \%)$ & $11(13.3 \%)$ & .494 \\
Operative procedure & $59.3 \pm 11.7$ & $60.4 \pm 13.1$ & .555 \\
AVR & & $13(15.7 \%)$ & .268 \\
MVR & $31(30.7 \%)$ & $22(26.5 \%)$ & .624 \\
MVP & $10(9.9 \%)$ & $9(10.8 \%)$ & .820 \\
CABG & & & .757 \\
\hline & & & \\
Aortic surgery & & & \\
\hline
\end{tabular}

Values are in mean \pm standard deviation [SD], number, number (\%)

Abbreviations: C, continuous use of fentanyl; S, single administration of fentanyl; M/F, male/female; ASA-PS, The American Society of Anesthesiologists physical status classification; AST, aspartate aminotransferase; ALT, alanine aminotransferase; EF, ejection fraction; AVR, aortic valve replacement; MVR, mitral valve replacement; MVP, mitral valvuloplasty; CABG, coronary artery bypass grafting

Table 2 Intraoperative profile of the patients 


\begin{tabular}{lccc}
\hline & S group & C group & p value \\
\hline Operative duration (min) & $336 \pm 87$ & $324 \pm 99$ & .168 \\
Anesthetic duration (min) & $423 \pm 99$ & $419 \pm 110$ & .357 \\
\hline Intraoperative rocuronium bromide dose $(\mathrm{mg})$ & $148 \pm 37$ & $139 \pm 40$ & .122 \\
\hline Intraoperative midazolam dose $(\mathrm{mg})$ & $7.6 \pm 2.1$ & $7.9 \pm 2.0$ & .254 \\
\hline Intraoperative fentanyl dose $(\mu \mathrm{g} / \mathrm{kg})$ & $27.8 \pm 10.1$ & $28.9 \pm 12.1$ & .494 \\
\hline Intraoperative remifentanil dose $(\mu \mathrm{g} / \mathrm{kg})$ & $0.71 \pm 0.89$ & $0.69 \pm 0.83$ & .880 \\
\hline RBC (ml) & $630 \pm 560$ & $588 \pm 518$ & .614 \\
\hline FFP (ml) & $648 \pm 528$ & $624 \pm 540$ & .823 \\
\hline PC (ml) & $178 \pm 230$ & $228 \pm 202$ & .113 \\
\hline Fluid volume (ml) & $3450 \pm 1118$ & $3518 \pm 1377$ & .714 \\
\hline$\quad$ Bleeding (ml) & $2096 \pm 925$ & $1962 \pm 477$ & .423 \\
\hline
\end{tabular}

Values are in mean \pm standard deviation $[\mathrm{SD}]$

Abbreviations: C, continuous use of fentanyl; S, single administration of fentanyl; RBC, red blood cell; FFP fresh frozen plasma; PC, platelet concentrate

Table 3 Postoperative profile of the patients 


\begin{tabular}{lccc}
\hline & S group & C group & p value \\
\hline Total DEX dose & $0.23 \pm 0.11$ & $0.24 \pm 0.10$ & .944 \\
Total time of DEX & $15.6 \pm 10.6$ & $16.2 \pm 9.5$ & .709 \\
\hline Total propofol dose (mg/kg/h) & $0.35 \pm 0.62$ & $0.37 \pm 0.55$ & .941 \\
\hline Total time of use of propofol (hr) & $3.5 \pm 6.8$ & $3.6 \pm 7.4$ & .935 \\
\hline Time to extubation (hr) & $7.0 \pm 5.3 \pm$ & $11.3 \pm 11.3$ & .0007 \\
\hline Total postsurgical dose of fentanyl ( $\mu$ g) & $42 \pm 120$ & $1060 \pm 228$ & $<.0001$ \\
\hline Total time of continuous use of fentanyl (hr) & - & $31.6 \pm 11.3$ & \\
\hline Total dose of acetaminophen (mg) & $804 \pm 926$ & $48.2 \pm 215.4$ & $<.0001$ \\
\hline P/F ratio & $282.2 \pm 53.4$ & $287.34 \pm 52.4$ & .515 \\
\hline PONV n (\%) & $5(5.0 \%)$ & $12(14.5 \%)$ & .0389 \\
\hline Constipation n (\%) & $48(47.5 \%)$ & $44(53.0 \%)$ & .554 \\
\hline AST (unit/L) & $37.4 \pm 16.7$ & $38.3 \pm 19.4$ & .739 \\
\hline ALT (unit/L) & $23.7 \pm 10.0$ & $24.8 \pm 16.0$ & .583 \\
\hline Serum creatinine (mg/dL) & $0.85 \pm 0.55$ & $0.85 \pm 0.38$ & .975 \\
\hline Total length of ICU stay (days) & $4.4 \pm 1.5$ & $5.3 \pm 1.9$ & .0003 \\
\hline
\end{tabular}

Values are in mean \pm standard deviation [SD], number, number (\%)

Abbreviations: C, continuous use of fentanyl; S, single administration of fentanyl; DEX, dexmedetomidine; AST, aspartate aminotransferase; ALT, alanine aminotransferase; $\mathrm{P} / \mathrm{F}, \mathrm{PaO}_{2} / \mathrm{FiO}_{2} ; \mathrm{PONV}$, Postoperative Nausea and Vomiting; ICU, Intensive Care Unit

\section{Figures}




\section{Patients were screened for eligibility}

3 Aborted surgery

14 Met exclusion criteria

-2 ASA-PS 4

-7 History or current dialysis

-5 Surgery without a median sternotomy

\section{Patients were analyzed}

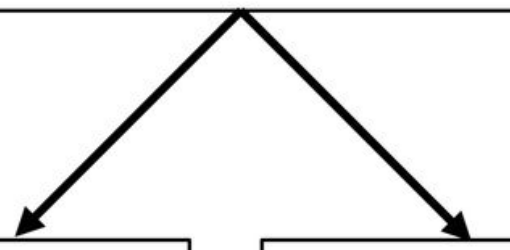

Single administration of fentanyl (Group S)

$\mathrm{N}=101$

Continuous administration of fentanyl (Group C)

$$
N=83
$$

\section{Figure 1}

Flowchart of patients of the retrospective study 


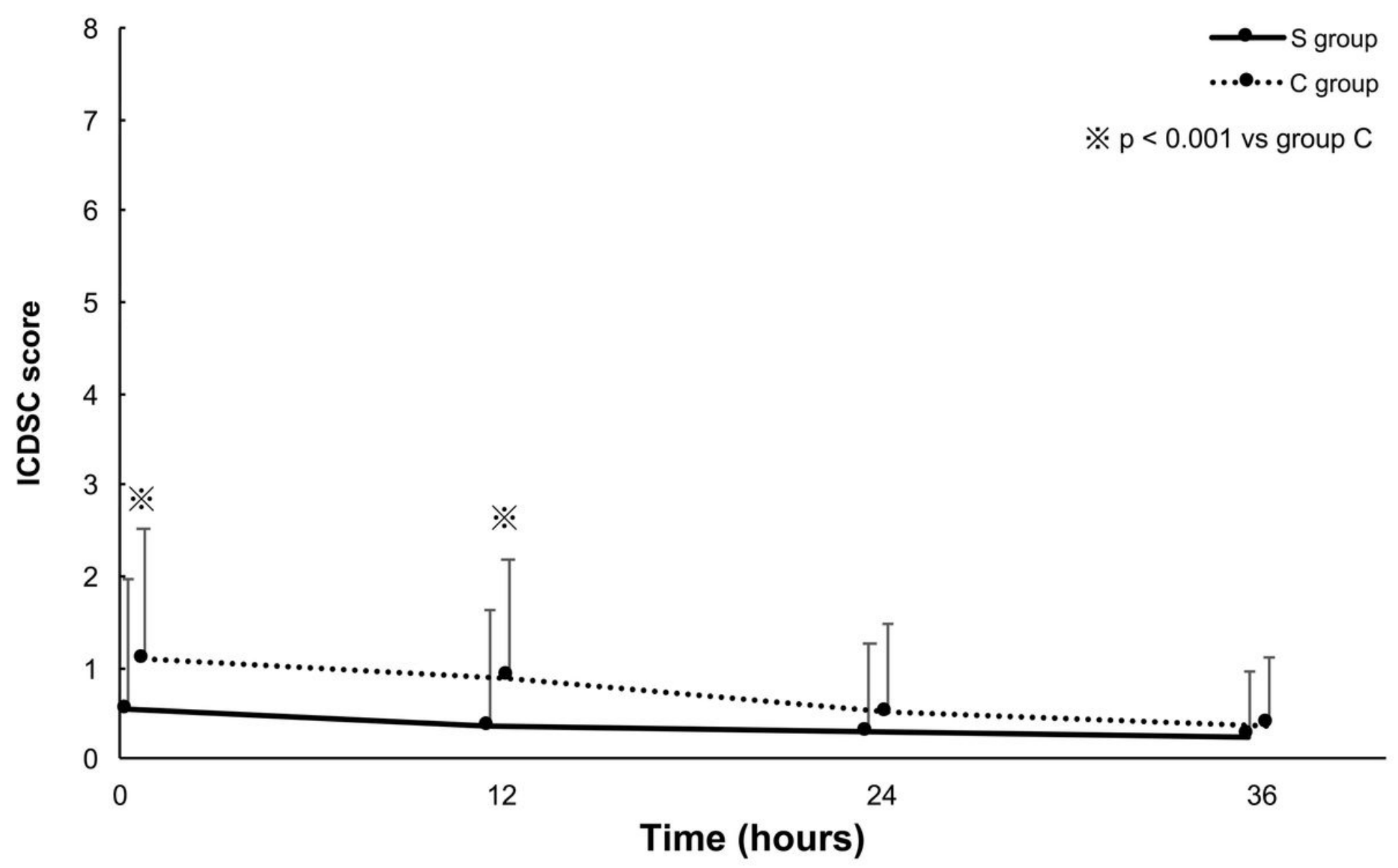

Figure 2

Change in ICDSC score after ICU admission Abbreviations: Group C, continuous administration of fentanyl group; Group S, a single administration of fentanyl group Values are represented as means \pm standard deviations [SDs] 


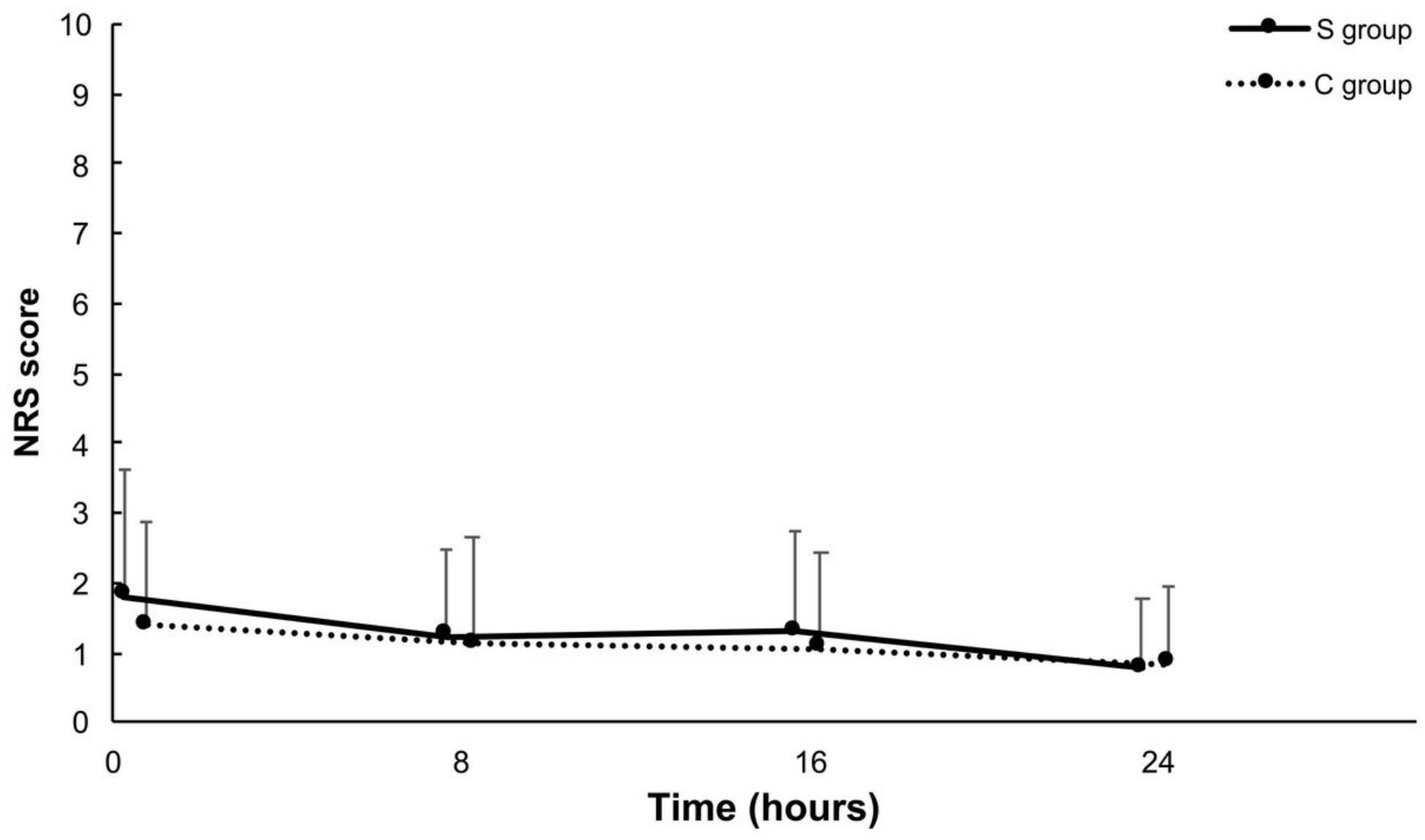

Figure 3

Changes in NRS scores after ICU admission Abbreviations: Group C, continuous administration of fentanyl group; Group S, a single administration of fentanyl group Values are represented as means \pm standard deviations [SDs] There were no statistically significant differences between the two groups.

\section{Supplementary Files}

This is a list of supplementary files associated with this preprint. Click to download.

- Additionalfile1.docx 